\title{
Reducing Health Disparities by Removing Cost, Access, and Knowledge Barriers
}

\author{
Melody Goodman, PhD; ${ }^{1}$ Ojiugo Onwumere; ${ }^{2}$ Laurel Milam, MA; ${ }^{1}$ \\ Jeffrey F. Peipert, MD, PhD; ${ }^{3}$
}

Division of Public Health Sciences; Departments of Surgery ${ }^{1}$ Washington University School of Medicine, St. Louis, Missouri;

Division of Clinical Research, Department of Obstetrics and Gynecology, ${ }^{2}$ Washington University School of Medicine, St. Louis, Missouri;

Department of Obstetrics and Gynecology, ${ }^{3}$ Indiana University School of Medicine; Indianapolis, Indiana.

\section{Address correspondence to:}

\author{
Jeffrey F. Peipert, MD, PhD \\ Clarence E. Ehrlich Professor and Chair \\ Department of Obstetrics and Gynecology \\ Indiana University School of Medicine \\ 550 N University Blvd., UH 2440 \\ Indianapolis, Indiana 46202 \\ Email: JPeipert@iu.edu \\ Phone: $\quad 317-944-8609$ \\ Fax: $\quad 317-944-7417$
}

Disclosures: J.F.P. receives research funding from Bayer, Teva and Merck and serves on an advisory boards for Teva and Perrigo.

Funding: The Contraceptive CHOICE Project was funded by an anonymous foundation.

This is the author's manuscript of the article published in final edited form as:

Goodman, M., Onwumere, O., Milam, L., \& Peipert, J. F. (2017). Reducing health disparities by removing cost, access, and knowledge barriers. American Journal of Obstetrics and Gynecology, 216(4), 382.e1-382.e5. 


\section{CONDENSATION}

Black-white health disparities in the rate of unintended pregnancies can be reduced by providing education, reducing access barriers, and providing contraception at no cost. 


\section{ABSTRACT:}

Importance: While the rate of unintended pregnancy has declined in the U.S. in recent years, unintended pregnancy among teens in the U.S. is the highest among industrialized nations, and disproportionately affects minority teens. Our objective of this secondary analysis was to estimate the risk of unintended pregnancy for both Black and White women age 15- 19 years when barriers to access, cost, and knowledge are removed. Our hypothesis was that the Black-White disparities would be reduced when access, education, and cost barriers are removed.

Design, Setting, and Participants: We performed an analysis of Contraceptive CHOICE Project (CHOICE) database. CHOICE is a longitudinal cohort study of 9,256 sexually active women ages $14-45$ in the St. Louis region from 2007 to 2013 . Two measures of disparities were used to analyze teenage pregnancy rates and pregnancy risk from 2008 to 2013 among teens ages 15-19. These rates were then compared to the rates of pregnancy among all sexually active teens in the US during the years 2008 , 2009, 2010, and 2011. We estimated an absolute measure (rate difference (RD)) and a relative measure (rate ratio $(R R)$ ) to examine Black-White disparities in the rates of unintended pregnancy.

Results: While national rates of unintended pregnancy are decreasing, racial disparities in these rates persist. The Black-White rate difference dropped from 158.5 per 1,000 in 2008 to 120.1 per 1,000 in 2011 ; however, the relative ratio disparity decreased only from 2.6 to 2.5 , suggesting that Black sexually active teens in the U.S. have 2.5 times the rate of unintended pregnancy as White teenagers. In the CHOICE Project, there was a decreasing trend in racial disparities in unintended pregnancy rates 
among sexually active teens (age 15-19); 2008-2009 (RD=18.2; $R R=3.7), 2010-2011$ $(\mathrm{RD}=4.3 ; \mathrm{RR}=1.2)$ and $2013-2014(\mathrm{RD}=-1.5 ; \mathrm{RR}=1.0)$.

Conclusions: When barriers to cost, access, and knowledge were removed, such as in the Contraceptive CHOICE Project, Black-White disparities in unintended pregnancy rates among sexually active teens are reduced on both absolute and relative scales. The rate of unintended pregnancy was almost equal between Black and White women compared to large Black-White disparities on the national level.

Word Count: 343 


\section{INTRODUCTION}

Over the past several years, the US birth rate has declined steadily. However, a disproportionate number of those births occur among Black and Hispanic women. Of the 3 million unintended pregnancies that occur each year in the United States, Black women are three times more likely and Hispanic women are twice as likely to have an unintended pregnancy in comparison to white women ${ }^{1,2}$. The US teen pregnancy rate is among the highest in the developed world, and the risk of death associated with these pregnancies is one-third higher than women 20-24 years of age. ${ }^{3}$ Teenagers are at particularly high risk for unintended pregnancy as they experience increased barriers to accessing contraceptives such as financial constraints, misinformation about long-acting reversible contraception (LARC), and unclear legal frameworks surrounding confidentiality for minors ${ }^{4}$.

Many unintended pregnancies occur because of incorrect or inconsistent use of contraceptives, and non-use of contraception ${ }^{5}$. Incorrect, inconsistent and non-use of contraception are highest among non-Hispanic Black and Hispanic women when compared to other racial/ethnic groups ${ }^{6}$. However, high rates of unintended pregnancies among minorities cannot solely be attributed to contraceptive failure and non-use.

Health care disparities also contribute to the high rates of unintended pregnancy seen among Black and Hispanic women. Disparities in health care usually occur at three points: 1) exposures to stressors throughout the life course, 2) access to medical care, and 3) quality of care received. Addressing the social determinants of health disparities by eliminating access, education, and cost barriers have the potential to significantly reduce racial disparities in health outcomes ${ }^{7}$. Non-Hispanic Blacks and Hispanics are also more likely to have lower education levels, poor quality of education, 
lower income, and lack health insurance in comparison to whites ${ }^{8-10}$. This is significant as several studies using data from the National Survey of Family Growth 2006-2008 have shown income and education attainment to be significant predictors for unintended pregnancy ${ }^{1}$.

Removing barriers to the most effective forms of contraception could reduce unintended teen pregnancies, improve the reproductive health of non-Hispanic Black and Hispanic women, and promote educational and career advancement. The purpose of this study is to examine the effects that removing cost, access, and knowledge barriers would have on non-Hispanic Black-White disparities in rates of unintended pregnancy.

\section{METHODS}

The Contraceptive CHOICE Project is a prospective cohort study; 9,256 women were enrolled. The study sought to reduce unintended pregnancies among women ages 14 to 45 by informing and educating them about the most effective reversible methods of contraception: long-acting reversible contraceptives (LARC) including intrauterine devices (IUDs) and the contraceptive implant. Participants were recruited from medical clinics, study flyers, and newspaper ads. Participants were enrolled from August 2007 to September 2011, and were provided the contraceptive method of their choice at no cost for 2-3 years, depending on the date of enrollment. The Human Research Protection Office at Washington University in St. Louis School of Medicine approved the protocol of the CHOICE Project.

Women were eligible to participate in the CHOICE Project if they did not want to become pregnant within the coming year, were between 14-45 years of age, were 
sexually active with a male or were going to be sexually active in the next 6 months, lived in the St. Louis area, and spoke English or Spanish. Women who had undergone a sterilization procedure or hysterectomy were excluded from the study. Before enrolling in the study, all participants gave written informed consent to join the study. Women under the age of 18 gave written assent and a guardian or parent gave written consent. If minors sought contraception without parental knowledge, they could enroll using a waiver of parental consent.

All participants received contraceptive counseling that presented contraceptives in order of most to least effective. In addition, the counseling reviewed risks, side effects, and benefits associated with each method. Participants choose the appropriate method of contraception for themselves and were offered same day insertion (when applicable) unless the patient desired an IUD and currently had cervicitis or if pregnancy could not be ruled out.

Participants conducted telephone interviews with study staff at 3 and 6 months and every 6 months until completion of 2-3 years of follow-up, depending on when participants enrolled in the study. During these interviews, information regarding demographic characteristics, pregnancy outcomes, contraceptive method use and satisfaction, and reproductive history of participants was obtained. The participants selfreported their race and ethnicity during these interviews. All pregnancies were documented in a pregnancy log as well as the contraceptive method that the participant was using at the time of the pregnancy. In addition, if the outcome of the pregnancy was known at the time of the survey, this information was also documented.

Estimates for pregnancy rates among sexually active teens in the U.S. were calculated from the yearly birth rate, abortion rate, and miscarriage rate for each year 
from 2008 to 2011 by race for non-Hispanic Whites and Blacks ages 15-19. Birth rates were obtained from The National Vital Statistics reports, a project of the National Center for Health Statistics $(\mathrm{NCHS})^{11-13}$. The abortion rate comes from the statistics released by the Guttmacher Institute ${ }^{14}$. The number of miscarriages is estimated to be $20 \%$ of births and $10 \%$ of abortions (miscarriage rate $=$ birth rate $\times 0.2+$ abortion rate $\times 0.1) .{ }^{13}$ The pregnancy rate is the sum of the birth rate, abortion rate, and the miscarriage rate, expressed in pregnancies per 1,000 teens. Using data from the National Survey of Family Growth, we calculated the percent of teens 15-19 who had ever had sex by race to develop race-specific estimates. This percentage is multiplied by the number teens (15-19) of each race to estimate the number of sexually active teens by race. The number of sexually active teenagers in the United States is the denominator and the pregnancy rate is the numerator of the race specific estimates.

CHOICE estimates of pregnancy rates among sexually active teens are calculated by dividing the number of pregnancies by person-years contributed by participants aged 15-19 and multiplied by 1,000 to get rate per 1,000 sexually active teens. Given the low number of pregnancies in some years, two-year rates were calculated (2008-2009, 2010-2011, 2012-2013; Table 1). Details of the assessment of unintended pregnancies in CHOICE can be found in Secura et al. ${ }^{15}$

We examined Black-White disparities in teenage (ages 15-19) pregnancy rates in U.S. population estimates and CHOICE project estimates using two disparities measures: one absolute (rate difference) and one relative measure (rate ratio). Rate difference $(\mathrm{RD})$ is the absolute difference between the unintended teenage pregnancy rates for Blacks $\left(r_{B}\right)$ and Whites $\left(r_{W}\right)$ and is calculated as $r_{B}-r_{W}$. The rate ratio (RR) is 
the relative difference and is calculated as $r_{B} / r_{w}$. RR measures the relative difference in the rates of the best and worst group at each time point.

\section{RESULTS}

We noted a decline in national teenage (ages 15-19) pregnancy rates among non-Hispanic Whites and Blacks between 2008-2011. While decreases in teenage pregnancy rates occur in both racial groups, larger decreases are seen among whites. The Black-White rate difference drops from 158.5 per 1,000 in 2008 to 120.1 per 1,000 teens in 2011 (Figure 1). This is a 38.4 per 1,000 decrease in the rate difference over the four-year period. The decrease in absolute racial disparity does not hold for relative racial disparity; the rate ratio decreases only slightly from 2.6 to 2.5 and then holds steady over the four-year period, suggesting that on average in the United States Black sexually active teenagers (15-19) have 2.5 times the rate of unintended pregnancy than White teenagers (Figure 1).

The CHOICE Project followed 1371 teens aged 15 to 19 over the study period. The majority of teens were non-Hispanic Black (61\%, 838 of 1371), and 28\% (384 of 1371) were non-Hispanic White. For 2008-2009, the unintended pregnancy rate in Black teenagers was 25.1 per 1,000 women-years, and in Whites 6.8 per 1,000 women years (Table 1). Using the two-year estimates, we found that the 2008 and 2009 period had the largest Black-White disparities $(R D=18.2 ; R R=3.7)$. For the subsequent twoyear period (2010-2011), both the absolute and relative measure decreased despite increases in unintended pregnancy rates in both groups $(R D=4.3 ; R R=1.2)$. For 20122013, the pregnancy rate for White teens in CHOICE exceeded that for Black teens by approximately 2 pregnancies per 1,000 person-years inverting both the rate difference 
and rate ratio $(R D=-1.5, R R=0.95)$, suggesting an elimination of racial disparities in unintended pregnancy. Figure 2 shows this reduction in racial disparities graphically. Using an absolute measure, there is a dramatic reduction in the Black-White disparity in teenage pregnancy rates in the CHOICE project when compared to the United States. Compared to the national rate is 158.5 in 2008 and 145.9 in 2009, the combined 20082009 RD among CHOICE participants is 18.2. Thus, not only are the teen pregnancy rates by race in $\mathrm{CHOICE}$ much lower than the national averages, the racial disparity (as an absolute measure) is also reduced (Figure 2).

When examining the relative disparity measures, the Black-White Rate Ratio in the CHOICE Project started out higher (2008-2009, RR=3.7) than the national average of 2.6 in 2008 and 2.5 in 2009 (Figure 1). However, by the following two-year period, we see reductions in the rate ratio $(2010-2011 \mathrm{RR}=1.2)$ and, by $2012-2013$, the BlackWhite relative disparity was essentially eliminated in CHOICE (RR=0.95; Figure 2). However, the change in the RR and RD for CHOICE between the two-year periods was not statistically significant.

\section{DISCUSSION}

CHOICE Participants, ages 15-19, who were offered free contraceptive access and tier-based counseling, had a much lower rate of unintended pregnancy, whether they were Black or White, than U.S. teens. In the CHOICE project, we see a disparities reduction of $>95 \%$ on the absolute scale and $>60 \%$ on the relative scale compared to national rates. In fact, improving access to contraceptives lowered the risk of 
pregnancy among Black and White women to approximately a risk ratio of 1 in comparison to the national ratio of 2.5 .

Providing free contraception and contraceptive counseling for women is essential in reducing the risk of pregnancy. A retrospective study conducted at HealthPartners, a nonprofit Minnesota health care organization, analyzed missed opportunities for pregnancy prevention. Investigators found that on average teens had 2.7 outpatient visits before they became pregnant, and that half of these visits represented missed opportunities to provide contraceptive counseling to sexually active teens during preventative health visits ${ }^{16}$. Ensuring that sexually active teens are given accurate contraceptive counseling during routine clinic visits can better educate women to avoid unintended pregnancy and improve optimal birth spacing.

CHOICE USED tiered counseling, presenting contraceptive methods from most to least effective. Participants were introduced to all birth control methods, including the most effective reversible methods.. This may have helped reduced heath disparities related to differences in quality of care and subconscious stereotyping by health care professionals. A study analyzing recommendations for intrauterine contraception presented doctors and health care professionals with videos of women seeking contraception. The women were White, Black, or Latina and were portrayed as being of low SES and then as high SES. The study found that doctors were more likely to recommend IUC to Black patients in comparison to White women and were less likely to recommend IUC to women who appeared to low SES. ${ }^{17}$ These findings suggest that there are biases that may influence the recommendation of the clinician. Using the tiered counseling method would aid in eliminating such influences, because patients are introduced to all methods regardless of their demographic characteristics; this ensures 
that the patients' contraceptive method is chosen based on their preference instead of any possible clinician bias.

Additionally, data from the Guttmacher Institute and the National Family Growth Survey has shown that lower income women have the highest rates of unintended pregnancy. Women below the federal poverty line have unintended pregnancy rates of 137 per 1,000 women in comparison to women at the highest income level (26 per 1,000) which is almost a 5-fold increase. ${ }^{18}$ With regard to race and ethnicity, a disproportionate number of Blacks and Hispanics fall below the national poverty line, which may contribute to this demographic struggling to obtain contraception that could prevent unintended pregnancies. ${ }^{19}$ Poor and low income women are at high risk, and account for a disproportionate number of unintended pregnancies, births, and abortions in the US.1,19 Thus, increasing access to contraception for women who are poor, underinsured or uninsured is essential to decrease the rates of unintended pregnancies.

When Title X Family Planning agencies in Colorado received private funding to support the increase of LARC uptake and provide contraception at no cost, investigators noted a $42 \%$ reduction in teen abortions and $40 \%$ reduction in teen births ${ }^{20}$. Additionally, LARC usage increased among poor women from 1 in 170 to 1 in 15 women, and reached $95 \%$ of young low income women. Interventions such as the one in Colorado clearly show that funding programs that provide no-cost contraception and counseling can significantly impact rates of unintended pregnancy among all women, teens, and especially minorities who represent a larger portion of those living below the federal poverty line. Health disparities disproportionally affect historically disadvantaged populations such as Blacks and Hispanics. Implementing programs that account for disparities such as poverty and educational inequalities between different populations 
can be an effective measure to reduce these disparities, as seen by the CHOICE project and others like those implemented in Colorado. We noted decreasing disparities in CHOICE as we were able to recruit greater numbers of disadvantaged women over time, improving the precision of our estimate.[Spain et al.]"

Strengths of the Contraceptive Choice project design include a large study sample featuring a diverse group of women who varied by age, income, race, and ethnicity. In addition, the study had a high rate of follow-up among participants. However, the small number of pregnancies by race in some years caused instability in 1-year CHOICE estimates of unintended pregnancy. We used 2-year rates in our analysis which are more stable and compare this to most recent national data available (2008-2010). The scale at which the CHOICE project was able to provide no-cost access of contraceptives distinguishes the study.

Our secondary analysis has several limitations. One is generalizability: the sample group of women was located in the St. Louis region and may not be fully representative of the U.S. population. In addition, the participants in CHOICE were motivated to actively seek out contraception which may not be true for the general population. Moreover, a larger portion of women in the CHOICE project were African American, and low income, and high risk for unintended pregnancy than the general population. We also limited our analysis to non-Hispanic Black and White teens to create a more homogeneous comparison; Hispanic women were excluded as the sample was too small to draw meaningful conclusions. 


\section{Conclusions}

Removing barriers to contraception, providing all methods including the most effective contraceptive methods at no-cost, and providing informative counseling reduced the risk of unintended pregnancy among teens in the CHOICE study and reduced racial disparity in pregnancy rates. 


\section{References}

1. Finer LB, Zolna MR. Declines in Unintended Pregnancy in the United States, 2008-2011. New England Journal of Medicine. 2016; 374: 842-852.

2. Susan CA. Abortion and Women of Color: The Bigger Picture. The Guttmacher Report on Public Policy.11.

3. Sedgh G, Finer LB, Bankole A, Eilers MA, Singh S. Adolescent pregnancy, birth, and abortion rates across countries: levels and recent trends. J Adolesc Health. 2015;56(2):223-230.

4. Kumar N, Brown JD. Access Barriers to Long-Acting Reversible Contraceptives for Adolescents. J Adolesc Health. 2016;59(3):248-253.

5. Frost JJ, Darroch JE, Remez L. Improving contraceptive use in the United States. Issues Brief (Alan Guttmacher Inst). 2008(1):1-8.

6. $\quad$ Dehlendorf C, Park SY, Emeremni CA, Comer D, Vincett K, Borrero S. Racial/ethnic disparities in contraceptive use: variation by age and women's reproductive experiences. Am J Obstet Gynecol. 2014;210(6):526 e521-529.

7. Jones CP, Jones CY, Perry GS, Barclay G, Jones CA. Addressing the social determinants of children's health: a cliff analogy. J Health Care Poor Underserved. 2009;20(4 Suppl):1-12.

8. Understanding Racial and Ethnic Differences in Health in Late Life: A Research Agenda. Washington DC: National Academies; 2004.

9. Suzanne Macartney AB, Kayla Fontenot. Poverty Rates for Selected Detailed Raceand Hispanic Groups by State and Place:2007 HispanAmerican Community Survey Briefs. (Febuary 2013 ).

10. Susan Aud MAF, Angelina KewalRamani. Status and Trends in the Education of Racial and Ethnic Groups. 2010.

11. Martin JA, Hamilton BE, Sutton PD, Ventura SJ, Mathews TJ, Osterman MJ. Births: final data for 2008. Natl Vital Stat Rep. 2010;59(1):1, 3-71.

12. Martin JA, Hamilton BE, Ventura SJ, et al. Births: final data for 2009. Natl Vital Stat Rep. 2011;60(1):1-70.

13. Martin JA, Hamilton BE, Ventura SJ, Osterman MJ, Wilson EC, Mathews TJ. Births: final data for 2010. Natl Vital Stat Rep. 2012;61(1):1-72.

14. Kost K HS. U.S. Teenage Pregnancies, Births and Abortions, 2010: National and State Trends by Age, Race and Ethnicity. 2014.

15. Secura GM, Madden T, McNicholas C, et al. Provision of no-cost, long-acting contraception and teenage pregnancy. N Engl J Med. 2014;371(14):1316-1323.

16. Kharbanda EO, Stuck L, Molitor B, Nordin JD. Missed opportunities for pregnancy prevention among insured adolescents. JAMA Pediatr. 2014;168(12):e142809.

17. Dehlendorf C, Ruskin R, Grumbach K, et al. Recommendations for intrauterine contraception: a randomized trial of the effects of patients' race/ethnicity and socioeconomic status. Am J Obstet Gynecol. 2010;203(4):319 e311-318.

18. Guttmacher Institute. Unintended Pregnancy in the United States. March 2016. https://www.guttmacher.org/sites/default/files/pdfs/pubs/FB-UnintendedPregnancy-US.pdf Accessed November 28, 2016.

19. Macartney, S, Bishaw A, Fontenot K. Poverty Rates for Selected Detailed Race and Hispanic Groups by State and Place: 2007-2011, February 2013.

http://www.census.gov/content/dam/Census/library/publications/2013/acs/acsbr1 1-17.pdf. Accessed November 28, 2016. 
20. Ricketts S, Klingler G, Schwalberg R. Game change in Colorado: widespread use of long-acting reversible contraceptives and rapid decline in births among young, low-income women. Perspect Sex Reprod Health. 2014;46(3):125-132.

21. Spain JE, Peipert JF, Madden T, Allsworth JE, Secura GM. The Contraceptive $\mathrm{CHOICE}$ Project: recruiting women at highest risk for unintended pregnancy and sexually transmitted infection. 
Table 1: Black-White Pregnancy Rates among Sexually Active Teenagers in the United States (2008-2011) and CHOICE Participants Aged 15-19 (2008-2013)

\begin{tabular}{ccccc|ccc}
\hline & \multicolumn{3}{c}{ United States } & \multicolumn{3}{c}{ CHOICE Project } \\
\cline { 2 - 8 } & 2008 & 2009 & 2010 & 2011 & $2008-2009$ & $2010-2011$ & $2012-2013$ \\
\cline { 2 - 8 } White & 101.0 & 96.2 & 87.7 & 82.4 & 6.8 & 27.2 & 32.0 \\
Black & 259.5 & 242.1 & 220.8 & 202.5 & 25.1 & 31.5 & 30.5 \\
\hline
\end{tabular}


Figure 1: Black-White Risk Difference and Rate Ratio of Pregnancy Rates among

Sexually Active Teenagers in the United States (2008-2011)

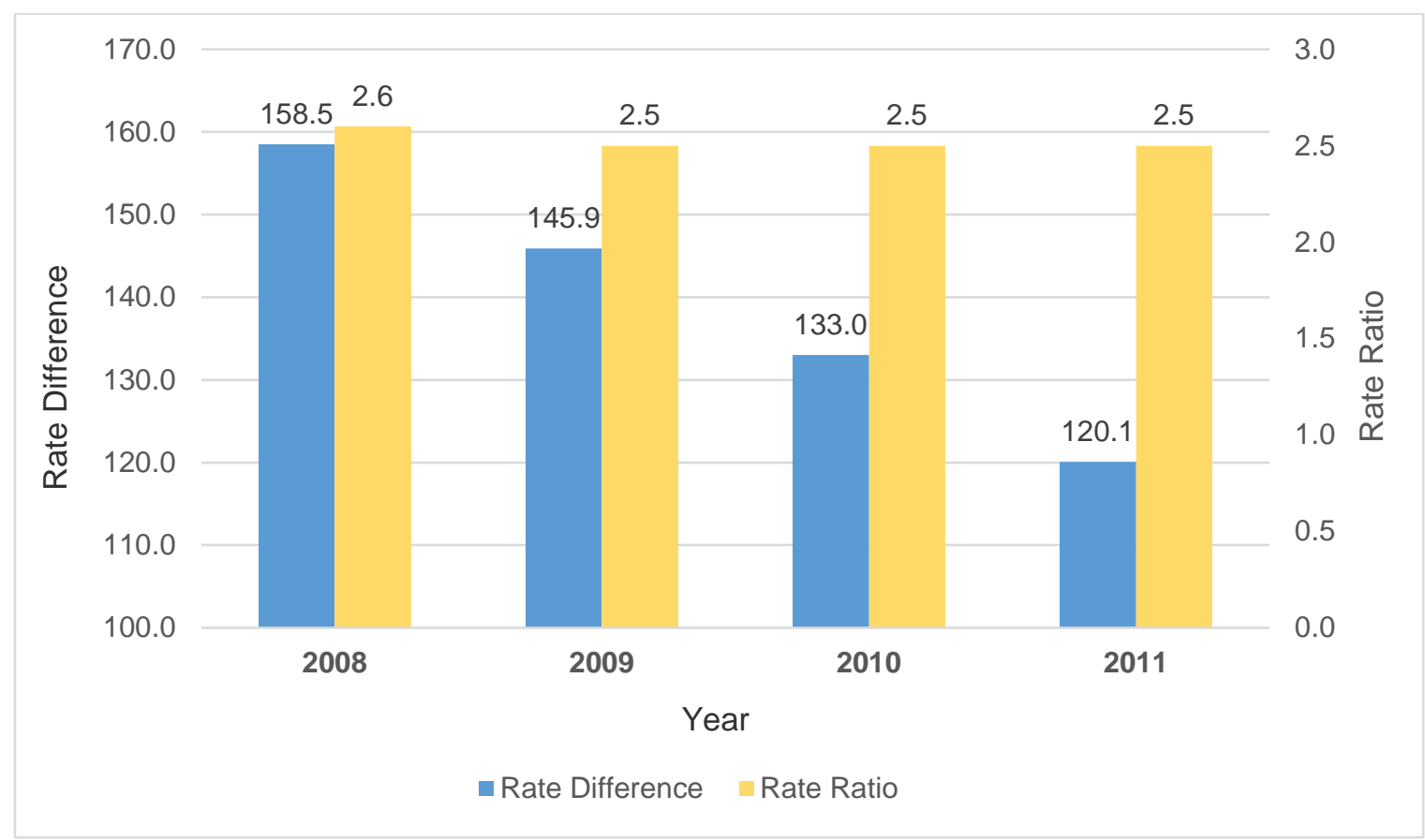


Figure 2: Black-White Risk Difference and Rate Ratio of Pregnancy Rates among CHOICE participants aged 15-19 (2008-2013)

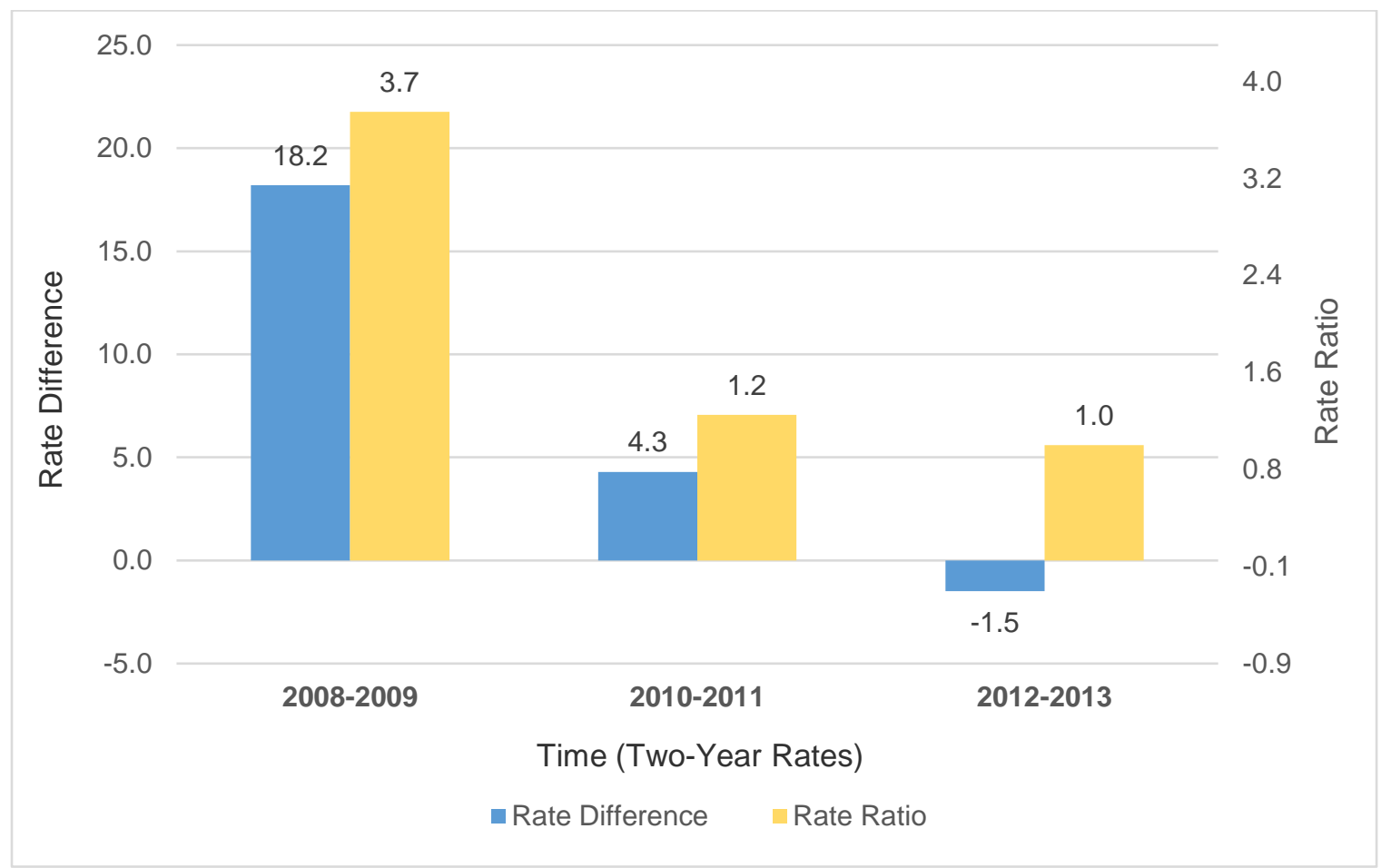

